Michele Silva de Barros

\title{
EFEITO DOS COMPONENTES SALIVARES DO MOSQUITO Aedes aegypti NA BIOLOGIA DE MACRÓFAGOS E POTENCIAIS APLICAÇÕES TERAPÊUTICAS
}

Tese apresentada ao Programa de Pós-Graduação em Imunologia do Instituto de Ciências Biomédicas da Universidade de São Paulo, para a obtenção do Título de Doutor em Ciências.

Área de concentração: Imunologia

Orientador: Prof. Dr. Anderson de Sá Nunes

Versão corrigida. A versão original eletrônica encontra-se disponível tanto na Biblioteca do ICB quanto na Biblioteca Digital de Teses e Dissertações da USP (BDTD). 


\section{RESUMO}

BARROS, M. S. Efeito dos componentes salivares do mosquito Aedes aegypti na biologia de macrófagos e potenciais aplicações terapêuticas. 2017. $107 \mathrm{f}$. Tese (Doutorado em Imunologia) - Instituto de Ciências Biomédicas, Universidade de São Paulo, 2017.

Os macrófagos são células fagocíticas derivadas dos monócitos sanguíneos produzidos pela medula óssea, mas que também podem se desenvolver a partir de progenitores embrionários com a capacidade de se renovar de maneira independente das células tronco hematopoiéticas. Os macrófagos estão diretamente envolvidos em um conjunto de processos biológicos vitais que inclui a eliminação de patógenos, a ativação de respostas imunes antígeno-específicas, o reparo tecidual e a regulação de processos inflamatórios, autoimunes e apoptóticos. Durante o repasto sanguíneo, fêmeas do mosquito Aedes aegypti inoculam saliva na pele de seu hospedeiro vertebrado e devido a sua localização estratégica nesse tecido, os macrófagos estão, possivelmente, dentre as primeiras células residentes do tecido a serem expostas a saliva. Porém, apesar dessa evidência fisiológica, sabe-se muito pouco sobre os efeitos imunomoduladores da saliva desse mosquito vetor sobre os macrófagos. Assim, o objetivo deste projeto foi avaliar o papel dos componentes salivares de A. aegypti na ativação de macrófagos peritoneais murinos e seu potencial efeito no desenvolvimento da encefalomielite autoimune experimental (EAE), um modelo murino para estudo da esclerose múltipla humana. Nossos resultados mostram que macrófagos peritoneais elicitados por tioglicolato e estimulados por LPS e IFN- $\gamma$ produzem menos óxido nítrico (NO), IL-6 e IL-12p70 e mais IL-10 quando pré-incubados com o extrato de glândula salivar (EGS) do mosquito. Observamos que a diminuição na produção dos mediadores inflamatórios estudados não parece ser devido a indução de morte dos macrófagos, pois não foram observadas alterações no metabolismo celular e nem um aumento significativo na exposição de fosfatidilserina na superfície deste tipo celular quando incubado com EGS de A. aegypti. Em nossas condições experimentais, observamos que a pré-incubação com EGS é também capaz de diminuir a

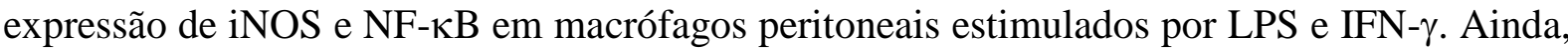
vimos que o EGS interfere negativamente na polarização para o perfil M1, mas não parece afetar a polarização para o perfil M2 nessas células. O fracionamento do EGS total por cromatografia líquida de alta eficiência (HPLC) revelou uma fração que reproduziu o fenótipo de inibição da produção de IL-6 e a análise por espectrometria de massa identificou três possíveis proteínas candidatas associadas com essa atividade. Uma dessas proteínas foi testada no modelo de EAE, sendo capaz de diminuir significativamente os sinais clínicos, retardar o início da doença e reduzir sua incidência nos animais. Esse fenótipo parece ser uma consequência da imunomodulação do perfil de resposta típico da doença, uma vez que as células totais de baço do grupo co-inoculado com a proteína salivar no momento da indução da doença, produziram menos IFN- $\gamma$ (perfil Th1) e IL-17 (perfil Th17) antígeno-específicos em comparação com as células do grupo que recebeu apenas o veículo no momento da indução. Além disso, não foram observadas alterações na produção das citocinas IL-4 e IL-5 (perfil Th2) entre os dois grupos experimentais. Em conclusão, o EGS de A. aegypti apresenta efeito imunomodulador sobre macrófagos peritoneais estimulados com LPS e IFN- $\gamma$ e uma das proteínas potencialmente envolvidas nessa atividade foi capaz de afetar a polarização das respostas Th1 e Th17 associadas com o desenvolvimento da EAE, melhorando assim os sinais clínicos da doença.

Palavras-chave: Aedes aegypti. Macrófagos. Extrato da glândula salivar. Encefalomielite autoimune experimental. Imunomodulação. 


\begin{abstract}
BARROS, M. S. Effects of Aedes aegypti salivary components on the biology of macrophages and potential therapeutic applications. 2017. $107 \mathrm{p}$. Ph. D. thesis (Immunology) - Instituto de Ciências Biomédicas, Universidade de São Paulo, 2017.
\end{abstract}

Macrophages are phagocytic cells derived from blood monocytes produced by the bone marrow, but that can also develop from embryonic progenitors with the ability to renew themselves independently on hematopoietic stem cells. Macrophages are directly engaged in a number of vital biological processes including the elimination of pathogens, activation of antigen-specific immune responses, tissue repair and regulation of inflammatory, autoimmune and apoptotic processes. During blood feeding, Aedes aegypti female mosquitoes inoculate saliva into the skin of their vertebrate hosts and due to its strategic location in this tissue, macrophages are possibly among the first resident cells to be exposed to saliva. However, despite this physiological evidence, little is known about the immunomodulatory effects of $A$. aegypti saliva on macrophages. Thus, the aim of this project was to evaluate the role of $A$. aegypti salivary components on the activation of peritoneal murine macrophages and their potential effect on the development of experimental autoimmune encephalomyelitis (EAE), a murine model for studying human multiple sclerosis. Our results show that thioglycollateelicited peritoneal macrophages activated by LPS and IFN- $\gamma$ produce less nitric oxide (NO), IL-6 and IL-12p70 and more IL-10 when preincubated with the mosquito's salivary gland extract (SGE). The decreased production of the inflammatory mediators evaluated does not seem to be due to the induction of macrophages death, since neither changes in cell metabolism nor a significant increase in phosphatidylserine exposure on the surface on this cell type when incubated with SGE were observed. In our experimental conditions, we observed that pre-incubation with SGE is also able to decrease iNOS and NF- $\kappa \mathrm{B}$ expression on peritoneal macrophages stimulated by LPS and IFN- $\gamma$. Also, we have seen that the SGE negatively interferes with the polarization to the M1 profile, but does not affect the polarization to the M2 profile in these cells. SGE fractionation by high performance liquid chromatography (HPLC) revealed a fraction that reproduced the inhibitory phenotype on the IL-6 production, and the mass spectrometry analysis identified three putative proteins associated to this activity. One of these proteins was tested in the EAE model, being able to significantly decrease the clinical signs, delay the disease onset and reduce the incidence of the disease in the animals. This phenotype seems to be a consequence of the immumodulation of the disease's typical response pattern, since the total spleen cells of the group co-inoculated with the salivary protein during the disease induction, produced less antigen-specific IFN- $\gamma$ (Th1 pattern) and IL-17 (Th17 pattern) in comparison to the cells of the group receiving only the vehicle. In addition, no changes were observed in the production of the cytokines IL- 4 and IL-5 (Th2 pattern) between the two experimental groups. In conclusion, A. aegypti SGE presents an immunomodulatory effect on peritoneal macrophages stimulated by LPS/IFN- $\gamma$ and one of the proteins potentially involved in this activity was capable of affecting the polarization of Th1 and Th17 responses associated to the EAE development, thus improving the clinical signs of the disease.

Keywords: Aedes aegypti. Macrophages. Salivary gland extract. Experimental autoimmune encephalomyelitis. Immunomodulation. 


\section{INTRODUÇÃO}

\subsection{Macrófagos}

No final do século 19, o zoólogo russo Ilya Ilyich Mechnikov (Élie Metchnikoff) mostrou que quando espinhos de tangerineira eram introduzidos em larvas de estrelas-do-mar, eles ficavam recobertos por células móveis que foram denominadas "fagócitos". A partir dessa descoberta, Metchnikoff observou a existência de células semelhantes em uma série de outros organismos e, naqueles com um sistema vascular sanguíneo, os fagócitos eram os leucócitos que "escapavam" dos vasos sanguíneos durante a inflamação e que possuíam a capacidade de digerir bactérias. Em uma de suas mais importantes obras, "Immunity in infective diseases" de 1905, Metchnikoff usou o termo "macrófagos" para diferenciar leucócitos mononucleares maiores e com capacidade de realizar fagocitose, daqueles menores e que claramente não possuíam capacidade fagocítica (linfócitos). Esta foi a base de sua “Teoria da Imunidade Celular", cujos estudos foram reconhecidos com o Prêmio Nobel em $\begin{array}{lllll}\text { Fisiologia } & \text { ou } & \text { Medicina } & \text { de }\end{array}$ (http://www.nobelprize.org/nobel_prizes/medicine/laureates/1908/\#). Em 1924, o termo macrófagos foi utilizado por Karl Albert Ludwig Aschoff para nomear um conjunto de células do sistema reticuloendotelial composto não apenas por monócitos, macrófagos e histiócitos, mas também por fibroblastos e células endoteliais e reticulares. Mais tarde, em 1969, o conceito de sistema reticuloendotelial, que abrangia diferentes células no sentido funcional e imunológico, foi substituído pelo conceito de sistema mononuclear fagocítico que tinha como premissa que todos os macrófagos são derivados de monócitos sanguíneos. (SIEWEKE e ALLEN, 2013; ARANGO DUQUE e DESCOTEAUX, 2014; GUILLIAMS et al., 2014). Dessa forma, esse sistema é composto por um subgrupo de leucócitos originalmente descrito como uma população de células mieloides derivadas da medula óssea que circulam no sangue como monócitos e povoam tecidos como macrófagos no estado estacionário e durante a inflamação (VAN FURTH e COHN, 1968).

Metchnikoff foi o primeiro cientista a apreciar plenamente as capacidades e o propósito da fagocitose e reconhecer as diversas tarefas atribuídas a este processo. Como embriologista, observou a absorção de tecido durante a embriogênese, como zoologista, notou um mecanismo comum de alimentação de organismos unicelulares e, como patologista, observou seu importante papel na defesa do hospedeiro (YONA e GORDON, 2015). Ele observou que a fagocitose é mais do que a capacidade de uma célula englobar 
micro-organismos estranhos, ela também representa um mecanismo de defesa ativo e essa percepção levou ao conceito de imunidade inata (GUILLIAMS et al., 2014). Desde as descobertas de Metchnikoff, os imunologistas e biologistas celulares se ocuparam com o conceito de que os macrófagos são células imunes efetoras e com a compreensão de como estas células participam na defesa do hospedeiro. Essa abordagem se consolidou entre as décadas de 1960 e 1980 com a demonstração de que essas células podem ser "ativadas" (MACKANESS, 1962; 1964) e que essa ativação poderia ser realizada pelos linfócitos (NATHAN et al., 1971) através de uma citocina denominada interferon- $\gamma$ (NATHAN et al., 1983). No entanto, ao se concentrar sobre as funções imunológicas dessas células, durante muito tempo foi ignorado seu papel vital na homeostasia, que é independente da sua participação nas respostas imunes (MOSSER e EDWARDS, 2008).

Até pouco tempo, acreditava-se que a grande maioria dos macrófagos era derivada de células precursoras da medula óssea que se desenvolveram em monócitos. Os monócitos, por sua vez, se desenvolvem neste órgão hematopoiético à partir de células tronco da linhagem granulocítica-monocítica que foram expostas ao fator estimulador de colônias de macrófagos e granulócitos (GM-CSF) e a interleucina-3 (IL-3) (ARANGO DUQUE e DESCOTEAUX, 2014). Porém, dados recentes contrapõem a visão centrada e exclusiva dos monócitos como origem dos macrófagos. Isso porque foi descoberto que as principais populações de macrófagos são derivadas de progenitores embrionários que tem a capacidade de se renovar independente das células tronco hematopoiéticas. Nesse processo, não é necessário haver progenitores, pois os macrófagos maduros podem proliferar em resposta a estímulos específicos e sem transformação ou perda de diferenciação funcional, sugerindo assim que os macrófagos são células maduras diferenciadas que podem ter um potencial de autorrenovação semelhante ao das células-tronco. No entanto, muitas perguntas ainda precisam ser respondidas sobre esse potencial proliferativo e seus efeitos na homeostase e inflamação (SIEWEKE e ALLEN, 2013).

Em relação aos monócitos, sabe-se que os mesmos são liberados para a circulação e, em poucos dias, distribuem-se por todo o organismo. Em adultos saudáveis, a meia-vida dos monócitos circulantes é de aproximadamente 70 horas e essas células constituem cerca de 1-6\% dos leucócitos totais no sangue periférico. Quando os monócitos migram da circulação para os tecidos, extravasando através do endotélio da microcirculação, eles se diferenciam em macrófagos e células dendríticas (DCs) inflamatórias. O processo de diferenciação de monócitos em macrófagos envolve muitas alterações como o aumento do tamanho da célula 
de 5 a 10 vezes, aumento das organelas em número e complexidade e aumento da capacidade fagocítica. Dentre os fagócitos mononucleares, frequentemente, os macrófagos são distinguidos das DCs pela expressão diferencial de marcadores de superfície como o F4/80, CD11b e CD18 (também conhecido como MAC1), CD68 e receptores para a porção Fc dos anticorpos. Porém, poucas combinações conhecidas desses marcadores podem separar em definitivo esses dois tipos celulares uma vez que essas populações provêm de um desenvolvimento contínuo de progenitores mieloides comuns (MURRAY e WYNN, 2011; SIEWEKE e ALLEN, 2013; ARANGO DUQUE e DESCOTEAUX, 2014).

Os macrófagos estão amplamente distribuídos pelo corpo e são divididos em subpopulações de acordo com sua localização anatômica e fenótipo funcional (GORDON e TAYLOR, 2005). Assim, macrófagos especializados residentes em tecidos incluem osteoclastos (ossos), macrófagos alveolares (pulmões), histiócitos (tecido conjuntivo intersticial) e células de Kupffer (no fígado). No intestino encontram-se vários tipos de macrófagos e DCs com funções e fenótipos diferentes trabalhando em conjunto para a manutenção da tolerância no local. Além disso, diferentes populações de macrófagos também são encontradas nos órgãos linfoides secundários como os macrófagos presentes na zona marginal do baço e no seio subcapsular dos linfonodos, com diversas funções já descritas nessas regiões. Ainda, subpopulações distintas de macrófagos também podem ser encontradas em locais denominados "sítios imunoprivilegiados" como o cérebro (micróglia), olhos e testículos (PLOWDEN et al., 2004; MURRAY e WYNN, 2011). A ampla distribuição dos macrófagos sugere uma função essencial e importante dessas células como sensor de sinais de perigo endógenos ou exógenos (PLOWDEN et al., 2004).

Embora os macrófagos sejam bem conhecidos por realizar a captura de agentes estranhos que entram no organismo como, por exemplo, micróbios e outros materiais particulados, há diversas funções homeostáticas também atribuídas a esses fagócitos mononucleares em quase todos os tecidos do corpo. Os macrófagos são capazes de remover cerca de $2 \times 10^{11}$ eritrócitos por dia em humanos, o que equivale a quase $3 \mathrm{~kg}$ de ferro e hemoglobina por ano que são "reciclados" para reuso do organismo. Este é um exemplo de uma contribuição metabólica vital dessas células sem a qual o organismo não poderia sobreviver (MOSSER e EDWARDS, 2008; SIEWEKE e ALLEN, 2013). Macrófagos teciduais apresentam um amplo papel na manutenção da homeostasia do tecido por meio da remoção de células senescentes ou apoptóticas e na remodelação e reparo de tecidos após uma lesão ou infecção (GORDON e TAYLOR, 2005; TAYLOR et al.., 2005; MOSSER e 
EDWARDS, 2008; SIEWEKE e ALLEN, 2013). Estes processos independem de uma sinalização imune celular e a remoção das células supracitadas parece resultar em pouca ou nenhuma produção de mediadores imunes. Isto ocorre porque, de um modo geral, os receptores envolvidos neste processo de remoção homeostático (receptores scavenger, receptor da fosfatidilserina, de trombospondina e receptores de integrinas e complemento) produzem sinais inibitórios ou citocinas/mediadores anti-inflamatórios que permitem que a fagocitose ocorra independente da participação de outras células do sistema imune. Assim, além de ser uma importante célula imune efetora, os macrófagos também funcionam como células "zeladoras" que tem como função principal remover o material extracelular estranho do ambiente intersticial (MOSSER e EDWARDS, 2008).

Como descrito acima, os macrófagos são células heterogêneas, presentes em diversos tecidos, onde são responsáveis por processos inflamatórios, imunológicos e metabólicos (PLOWDEN et al., 2004). No processo inflamatório, os macrófagos apresentam três funções principais: fagocitose, apresentação de antígenos e imunomodulação através da produção de citocinas e fatores de crescimento (FUJIWARA e KOBAYASHI, 2005). A inflamação possui componentes da resposta inata e da resposta adaptativa, sendo desencadeada por estímulos e condições nocivas, tais como infecção e lesão tecidual (MEDZHITOV, 2008). O processo inflamatório é geralmente bem regulado e envolve tanto os sinais que iniciam e mantêm a inflamação quanto os sinais que finalizam este processo. No entanto, um desequilíbrio entre esses sinais pode causar um processo inflamatório descontrolado que resulta em dano celular e tecidual. Neste contexto, os macrófagos desempenham um papel crítico na iniciação, resolução e manutenção da inflamação, sendo ativados e desativados durante o processo inflamatório. Sinais de ativação incluem citocinas (IFN- $\gamma$, GM-CSF e TNF- $\alpha$, dentre outras), lipopolissacarídeos (LPS) bacterianos, proteínas da matriz extracelular e mediadores lipídicos, dentre outros. A inibição da inflamação pela remoção ou regulação de mediadores inflamatórios e células efetoras permite ao hospedeiro reparar os danos teciduais. É importante notar que os macrófagos desempenham um papel chave neste processo de regulação negativa (FUJIWARA e KOBAYASHI, 2005).

Os macrófagos apresentam um papel central na inflamação, pois iniciam a resposta imune contra micro-organismos uma vez que estão entre as primeiras células a entrar em contato com os agentes invasores. Esse fato se deve, em parte, pelo seu repertório de receptores do tipo Toll e scavenger que apresentam uma ampla capacidade de ligação a lectinas, lipoproteínas, proteínas, oligonucleotideos e polissacarídeos, entre outros. Além 
disso, macrófagos também expressam moléculas do complexo principal de histocompatibilidade (MHC) de classe II na sua membrana, o que possibilita a apresentação de antígenos extracelulares para linfócitos após captura e processamento dos mesmos. Após esse reconhecimento, os linfócitos $\mathrm{T}$ produzem citocinas capazes de ativar linfócitos $\mathrm{B}$ que, quando ativados, produzem anticorpos específicos para o antígeno apresentado pelo macrófago. Esses anticorpos ligam-se a antígenos nos micróbios ou nas células por eles invadidas e esse complexo antígeno-anticorpo é fagocitado mais avidamente por macrófagos (ARANGO DUQUE e DESCOTEAUX, 2014). Os macrófagos não só possuem uma ampla variedade de receptores de superfície celular e mediadores intracelulares que permitem o reconhecimento, fagocitose e destruição de patógenos, como também são equipados com moléculas secretoras essenciais que servem para regular as atividades das células vizinhas (VAN DYKEN e LOCKSLEY, 2013). Em resposta a patógenos e sinais de "dano/perigo", os macrófagos são capazes de secretar uma grande variedade de citocinas como, por exemplo, IL-1，IL-6，IL-10，IL-12，IL-15，IL-18，TNF- $\alpha ， I F N-\alpha$ e $\gamma$, TGF- $\beta$, GM-CSF，M-CSF, quimiocinas como CXCL8 (IL-8), CXCL1 - 3 (GRO $\alpha, \beta$ e $\gamma$, respectivamente), CXCL10 (IP-10) e CXCL5 (ENA-78), fatores de coagulação, prostaglandina $\mathrm{E}_{2}\left(\mathrm{PGE}_{2}\right)$, leucotrienos, intermediários reativos de oxigênio e nitrogênio, componentes do complemento, fatores angiogênicos e várias enzimas e proteases. A secreção desses fatores depende do estímulo e da localização dos macrófagos. Desta forma, os macrófagos também criam e modulam um ambiente favorável para respostas imunes adaptativas (PLOWDEN et al., 2004).

Mais recentemente, subtipos de macrófagos com funções distintas foram descritos. Os macrófagos M1 ("classicamente ativados") participam da defesa contra uma grande variedade de bactérias, protozoários e vírus e também apresentam um papel na imunidade antitumoral. Já os macrófagos M2 (“alternativamente ativados") apresentam funções anti-inflamatórias e regulam a cicatrização de feridas. Esses macrófagos "reguladores" produzem uma grande quantidade de IL-10 em resposta a ativação de seus receptores Fcy e scavenger (VARADHACHARY et al., 2001; MURRAY e WYNN, 2011). Macrófagos associados a tumores (TAMs) suprimem a imunidade antitumoral e células supressoras derivadas da linhagem mielóide (MDSCs) estão ligadas a TAMs, podendo inclusive ser suas precursoras. Dentre os cinco subtipos celulares descrito acima, quatro deles (macrófagos M2, macrófagos reguladores, TAM e MDSC) exibem atividade imunossupressora (MURRAY e WYNN, 2011). 
Como visto, os macrófagos são células bastante importantes dentro do sistema imune e trabalham de maneira integrada com diversas outras células que compõem esse sistema. $\mathrm{O}$ bom desempenho dessas células é de extrema importância para a manutenção de um organismo saudável. Assim, o entendimento sobre a modulação das funções dos macrófagos por diversas moléculas e substâncias da biodiversidade, incluindo extrato de plantas e produtos derivados de organismos, como salivas e venenos, por exemplo, pode servir como ferramentas de estudo para entender a biologia dessas células e também servir como fonte para a descoberta de novas substâncias com potencial terapêutico.

\subsection{Efeito da saliva de artrópodes hematófagos na hemostasia e em macrófagos}

Os artrópodes hematófagos são responsáveis pela transmissão de diversas doenças humanas e veterinárias, transmissão essa que ocorre no momento da alimentação sanguínea desses organismos. Para a obtenção do sangue em estado fluído, os artrópodes hematófagos precisam neutralizar a hemostasia do hospedeiro, que inclui os processos de agregação plaquetária, coagulação sanguínea e vasoconstrição. No contexto do repasto sanguíneo, a saliva torna-se o elemento de interação entre os artrópodes hematófagos e seus hospedeiros vertebrados (FRANCISCHETTI et al., 2009; SÁ-NUNES e OLIVEIRA, 2009). Ao longo da coevolução entre esses organismos, uma série de moléculas salivares com ações imunofarmacológicas surgiram e foram selecionadas para facilitar essa interação e permitir que a alimentação ocorra com sucesso. Na saliva de artrópodes hematófagos existe uma série de componentes anticoagulantes, antiagregantes de plaquetas e vasodilatadores, capazes de contrapor os efeitos do repasto sanguíneo no sistema vascular (RIBEIRO, 1987; REUNALA et al., 1990; RIBEIRO, 2000). A agregação plaquetária é a primeira linha de defesa para evitar a perda de sangue após lesão do tecido. Nesse sentido, diversos fatores podem desencadear a agregação plaquetária como: a adenosina difosfato (ADP), que pode ter origem de células lesadas ou liberado por plaquetas ativadas, o colágeno presente nas superfícies endoteliais, a trombina que é produzida durante a coagulação do sangue também pode influenciar esse processo e o tromboxano $\mathrm{A}_{2}\left(\mathrm{TXA}_{2}\right)$ produzido por plaquetas ativadas (RIBEIRO et al., 2012). Um dos mecanismos mais utilizados pelos insetos hematófagos para bloquear a agregação plaquetária é a hidrolise do ADP. A atividade da enzima apirase encontrada na saliva de alguns mosquitos culmina na inibição da agregação plaquetária pela hidrólise de ATP (adenosina trifosfato) e ADP. Neste processo ocorre a liberação de AMP (adenosina 
monofosfato) e fosfato inorgânico que são incapazes de agregar plaquetas (RIBEIRO et al., 1984). Posteriormente essa enzima foi caracterizada como um membro da família de genes da 5'-nucleotidase (CHAMPAGNE et al., 1995). A aegyptina, um membro da família de alérgenos de $30 \mathrm{kDa}$, também exerce ação sobre a agregação plaquetária por ser capaz de se ligar especificamente ao colágeno e bloquear a agregação de plaquetas induzida por essa proteína. Além disso, essa proteína também inibe a interação do fator de von Willebrand ao colágeno do tipo III (CALVO et al., 2007). Os vasodilatadores são moléculas que aumentam o fluxo sanguíneo, antagonizando vasoconstritores produzidos pelo sistema hemostático após, por exemplo, lesão do tecido pelo aparelho bucal dos insetos. A vasodilatação observada durante o repasto sanguíneo deve-se a ação das sialocininas I e II, peptídeos da família das taquicininas. Foi descrito que as sialocininas agem diretamente sobre o endotélio ativando a produção de óxido nítrico, resultando na vasodilatação (RIBEIRO, 1992; CHAMPAGNE e RIBEIRO, 1994). O processo de coagulação pode ser iniciado tanto pela via de ativação do fator XII ou pela via do fator tecidual. O resultado levará a ativação do fator X ou Xa, que converte a protrombina em trombina e, por sua vez, cliva o fibrinogênio em fibrina, formando o coágulo de sangue. Após ativação, as plaquetas produzem o TXA 2 e também liberam serotonina e epinefrina, que são potentes vasoconstritores (RIBEIRO et al., 2012). O alvo dos componentes salivares para driblar a coagulação sanguínea são as proteases ou complexos envolvidos nesse processo, como a trombina e o fator Xa. Nesse contexto, foi descrito na saliva do Aedes aegypti um inibidor de serino-proteases (serpina) que atua sobre o fator Xa (AFXa - Anticoagulant-Factor Xa) com atividade anti-coagulante (STARK e JAMES, 1995; 1998).

Quando espécies hematófagas introduzem suas peças bucais na pele à procura de vasos sanguíneos, os componentes salivares inoculados também entram em contato com as diversas células residentes no tecido. Devido sua localização, os macrófagos estão dentre as primeiras células a entrar em contato tanto com a saliva injetada na pele do hospedeiro durante o repasto sanguíneo quanto com os patógenos carreados pela mesma (MCKIEL, 1959). Apesar dessa evidência fisiológica, muito pouco foi estudado até o momento sobre os efeitos imumoduladores da saliva de artrópodes hematófagos sobre os macrófagos em condições normais e na presença de micro-organismos. Um trabalho recente demonstrou que a saliva do carrapato Ixodes scapularis reduziu a produção de citocinas inflamatórias em macrófagos derivados de medula óssea, induzida por ligantes de receptores do tipo Toll (TLRs) e do tipo NOD (NLR) e também por bactérias da espécie Anaplasma 
phagocytophilum, transmitida por esse vetor (CHEN et al., 2012). Efeito semelhante foi observado quando a saliva de Ixodes ricinus foi incubada com macrófagos estimulados com IFN- $\gamma$ ou com espiroquetas da espécie Borrelia afzelii (KÝCKOVÁ e KOPECKÝ, 2006). Um imunossupressor descrito na saliva desta espécie (Iris - do inglês "Ixodes ricinus immunosuppressor") parece ser parcialmente responsável por essa atividade, uma vez que foi capaz de induzir fenótipo parecido em células mononucleares periféricas do sangue humano (LEBOULLE et al., 2002). Alguns trabalhos demonstraram que as atividades fagocítica e microbicida de macrófagos também são afetadas pela saliva de vetores. A saliva dos carrapatos Rhipicephalus sanguineus e I. ricinus bloqueou a atividade microbicida de macrófagos e inibiu a produção de óxido nítrico (NO) por essas células (FERREIRA e SILVA, 1998; KUTHEJLOVÁ et al., 2001). De maneira semelhante, o extrato de glândula salivar (EGS) de espécies de flebotomíneos também é capaz de inibir a produção de NO em macrófagos murinos (WAITUMBI e WARBURG, 1998; NORSWORTHY et al., 2004). Camundongos inoculados com a saliva de Lutzomyia longipalpis recrutam um grande número de macrófagos em um modelo de bolsa de ar subcutânea, sugerindo que componentes do EGS desse flebotomíneo têm efeito inflamatório induzindo a migração de macrófagos (TEIXEIRA et al., 2005). Além disso, a saliva de flebotomíneos também inibe a apresentação de antígenos (Theodos e Titus, 1993) e a produção de TNF- $\alpha$, ao mesmo tempo que aumenta a produção de IL-6 e IL-10 e de AMP-cíclico em macrófagos (SOARES et al., 1998). Apenas dois trabalhos na literatura mostram a ação da saliva de mosquitos em macrófagos. Schneider et al. (2010) sugerem que o EGS de A. aegypti diminui significativamente a expressão de IFN- $\beta$ e NO sintase induzível em macrófagos, enquanto aumenta de maneira transiente a expressão de IL10, mostrando assim que a saliva do mosquito tem efeito sobre estas células. Além disso, os resultados apresentados na dissertação de WASSERMAN (2005) mostrou que a saliva do A. aegypti é capaz de diminuir a produção de citocinas pró-inflamatórias como IL-1 $\alpha$, IL-6, IL-12 e TNF- $\alpha$ produzidas por macrófagos murinos da linhagem J744 estimulados com LPS (WASSERMAN, 2005). Em nosso conhecimento, até o momento nenhum trabalho avaliou o papel dos componentes salivares dos outros dois gêneros de mosquitos com relevância clínica, Culex e Anopheles, na biologia dos macrófagos. Estudos nessa área poderão contribuir para o entendimento dos efeitos da saliva no processo inflamatório no contexto do repasto sanguíneo e da transmissão de micro-organismos por esses vetores. Além disso, como os macrófagos produzem uma grande variedade de moléculas biologicamente ativas que participam das diversas etapas do processo inflamatório e imune, intervenções terapêuticas 
dirigidas aos macrófagos e aos seus produtos podem abrir novas vias para o controle de doenças inflamatórias e autoimunes como, por exemplo, a esclerose múltipla (EM).

\subsection{Esclerose múltipla e Encefalomielite Autoimune Experimental}

A EM é uma das doenças crônicas mais comuns e incapacitantes do sistema nervoso central (SNC). É caracterizada pela perda de oligodendrócitos, desmielinização e disfunção neurológica progressiva causada por uma resposta imune exacerbada, sendo uma das principais causas de incapacidade neurológica em adultos jovens, afetando mulheres mais frequentemente do que homens (HEMMER et al., 2002; MOLINÉ-VELÁZQUEZ et al., 2015). A EM pode ser desencadeada por fatores ambientais em indivíduos com perfis genéticos de risco e se desenvolve quando células $\mathrm{T}$ reagem contra proteínas presentes no SNC como, por exemplo, a mielina, resultando em um efeito secundário sobre os neurônios (COMPSTON e COLES, 2008; GOVERMAN, 2009). A destruição da bainha de mielina dos neurônios ocasiona um bloqueio do impulso elétrico em direção a célula alvo, resultando em diversos sintomas que variam desde distúrbios da fala até paralisia total (GOVERMAN, 2009). A EM tem sido cada vez mais estudada tanto na pesquisa básica quanto na clínica por ser uma doença debilitante, altamente complexa e de incidência relativamente elevada, embora haja grande variabilidade entre os diferentes países ao redor do mundo. Dados da Fundação de Esclerose Múltipla dos Estados Unidos mostram uma mediana global de prevalência da doença de 30 casos por 100 mil habitantes, mas países como Estados Unidos, Reino Unido e Alemanha apresentam mais de 100 casos por 100 mil habitantes, sendo que no Canadá esse número alcança 291 casos por 100 mil habitantes (PIETRANGELO e HIGUERA, 2015). No Brasil, segundo dados do Ministério da Saúde, a prevalência é de 15 casos para cada 100.000 habitantes (MINISTÉRIO-DA-SAÚDE, 2015).

A real etiologia da EM ainda é desconhecida, mas estudos indicam a importância do sistema imune na patogênese da doença influenciada por ambos fatores genéticos e ambientais. Os principais mecanismos que causam os danos e levam às manifestações clínicas são: inflamação, desmielinização e degeneração dos axônios (COMPSTON e COLES, 2008). Segundo Hemmer et al. (2002), independente do que inicie esse evento, dois passos são essenciais para induzir uma resposta imune no SNC: um meio pró-inflamatório que aumente a expressão de moléculas do MHC, de receptores co-estimulatórios e de citocinas inflamatórias e uma resposta imune adquirida ao antígeno. Antígenos liberados do SNC primam células T e B nos órgãos linfoides periféricos levando à ativação dessas células e subsequente geração de 
subpopulações autorreativas pró inflamatórias Th1 e Th17 (FROHMAN et al., 2006). Após expansão clonal, essas células infiltram o SNC por meio da interação de ligantes presentes na membrana das mesmas com moléculas de adesão expressas na superfície do endotélio do SNC. Juntamente com anticorpos, os linfócitos e outras células mononucleares rompem a barreira hematoencefálica com a ajuda de proteases e quimiocinas. Neste ambiente, as células reconhecem os antígenos alvo que geralmente são proteínas presentes na mielina. Células B se diferenciam em plasmócitos e produzem anticorpos específicos que se ligam a antígenos solúveis ou ligados a membrana das células. Células $\mathrm{T} \mathrm{CD}^{+}$específicas também infiltram o local causando lesão direta às células que apresentam antígenos via MHC de classe I, enquanto que as células T CD4 ${ }^{+}$Th1 e Th17 encontram antígenos apresentados via MHC de classe II. A reativação dessas células induz um grande aumento na produção de citocinas inflamatórias que atraem outras células imunes, como macrófagos, que contribuem para a inflamação por meio da liberação de mediadores imunológicos danosos e ataque fagocítico direto sobre a bainha de mielina, o que acaba por amplificar a resposta (HEMMER et al., 2002; WUEST et al., 2011; PRINEAS e PARRATT, 2012). A secreção de diversas citocinas inflamatórias como IL-12, IL-23, IFN- $\gamma$, além de proteases, anticorpos, óxido nítrico e glutamato, induzem dano na mielina e nos oligodendrócitos. Após essa variedade de mediadores inflamatórios serem secretados, células $\mathrm{T} \mathrm{CD}^{+}$do subtipo Th2 proliferam e começam a secretar citocinas como IL-4, IL-5 e IL-13 que desviam a resposta imune. Com o dano causado, a desmielinização pode bloquear ou dificultar a condução dos impulsos nervosos, fenômeno este que acaba resultando nos sintomas neurológicos apresentados pelos pacientes (BIELEKOVA et al., 2000; BIELEKOVA et al., 2006; KAUR et al., 2013; TULLMAN, 2013).

Muito do conhecimento sobre as alterações imunopatológicas na EM naturalmente adquirida surgiu a partir de modelos animais experimentais. Destes, a encefalomielite autoimune experimental (EAE) é o modelo mais antigo, melhor estabelecido e que reproduz grande parte dos aspectos clínicos, neuropatológicos e imunológicos da EM (HOHLFELD e WEKERLE, 2001). Mesmo com algumas limitações (WEKERLE et al., 2012), o modelo de EAE foi sendo refinado ao longo das últimas décadas, com o uso inicial de preparações de extrato total ou extrato da substância branca do cérebro, seguido pela mielina purificada e finalmente por peptídeos derivados da proteína básica de mielina (MBP) ou da glicoproteína da mielina de oligodendrócitos (MOG), preparadas em emulsões com adjuvante de Freund completo (BAXTER, 2007). Outras proteinas que não fazem parte da mielina também podem ser utilizadas para indução da EAE (KOJIMA et al., 1994; BERGER et al., 1997), embora 
nem todas as proteinas do SNC sejam capazes de induzir a doença (Määttä et al., 1998; Thoua et al., 2000). Esse modelo auxiliou na demonstração de que tanto os elementos do sistema imune inato quanto do sistema imune adquirido estão envolvidos na patogênese da doença. Embora por muitas décadas a vasta maioria dos estudos tenha investigado o papel de linfócitos T e B (BELL e PATERSON, 1960; WAKSMAN et al., 1961; GONATAS e HOWARD, 1974; ORTIZ-ORTIZ e WEIGLE, 1976; BEN-NUN et al., 1981; ZAMVIL e STEINMAN, 1990; KOJIMA et al., 1994; LITZENBURGER et al., 1998; LUCCHINETTI et al., 2000), hoje se sabe que as células apresentadoras de antígeno, tanto da periferia (como os macrófagos e as células dendríticas), quanto as do SNC (células microgliais), estão associadas ao processo de indução da doença, uma vez que apresentam antígenos aos linfócitos T, geram coestimulação e produzem citocinas requeridas para ativação e diferenciação dos linfócitos $\mathrm{T}$ em células efetoras (LINK et al., 1999; GRETER et al., 2005; ALMOLDA et al., 2011; CHASTAIN et al., 2011). Como resultado temos a desmielinização primária das trilhas axonais, falha na condução axonal no SNC e paralisia progressiva dos membros posteriores. Dessa forma, o modelo de EAE é um modelo bastante útil uma vez que muitas das patologias observadas no SNC de animais com EAE apresentam uma forte similaridade com aquelas encontradas no SNC dos pacientes com EM (HOHLFELD e WEKERLE, 2001; MANGIARDI et al., 2011; MCCARTHY et al., 2012; ROBINSON et al., 2014).

Os mecanismos celulares e moleculares envolvidos na patogênese da doença ainda não são completamente compreendidos, mas sabe-se que a infiltração de linfócitos CD4 ${ }^{+}$Th1 e Th17 contribuem significativamente para a inflamação e desmielinização observadas no SNC (MERRILL et al., 1992; DUONG et al., 1994; LANGRISH et al., 2005; PARK et al., 2005; KOMIYAMA et al., 2006; BATOULIS et al., 2011; BECHER e SEGAL, 2011). A participação das células do perfil Th17 parece ser mais importante que as células Th1 no desenvolvimento da EAE. Langrish et al. (2005) mostraram que a tranferências de células Th17 para animais suscetíveis levou a sintomas mais graves que a transferência de células Th1 e que uma melhora foi observada quando os animais foram submetidos ao tratamento com anticorpos anti-IL-17. Da mesma forma, sintomas menos severos também podem ser obsevados em animais que não produzem IL-17, nos quais a ausência dessa citocina levou a um atraso no início do desenvolvimento da doença, redução do escore máximo, melhora nas alterações histológicas e na recuperação (KOMIYAMA et al., 2006).

Dentre os diversos mediadores e mecanismos envolvidos na iniciação e desenvolvimento da EAE está a citocina IL-6 produzida pelos macrófagos, dentre outras 
células (DIAB et al., 1997; SAMOILOVA et al., 1998). A IL-6 é uma citocina pleiotrópica que apresenta tanto atividades pró-inflamatórias quanto anti-inflamatórias. Assim como o TNF- $\alpha$, a IL-6 é um pirógeno endógeno que induz febre e produção de proteínas de fase aguda no fígado. As propriedades pró-inflamatórias dessa citocina são observadas quando a sinalização ocorre via interação do receptor solúvel da IL-6 com a molécula gp130 que é amplamente expressa em todas as células. Esta via de sinalização induz o recrutamento de monócitos para o local inflamado, promove a manutenção de células Th17, além de inibir apoptose de células $\mathrm{T}$ e desenvolvimento de células $\mathrm{T}$ reguladoras. Já as propriedades antiinflamatórias são observadas quando a sinalização ocorre pela via clássica, na qual a citocina se liga ao seu receptor que é expresso em poucas células apenas (ARANGO DUQUE e DESCOTEAUX, 2014). Esta citocina, juntamente com o TGF- $\beta$, induz a diferenciação de linfócitos T CD4 ${ }^{+}$efetores para o perfil Th17 de resposta (KORN et al., 2008; KORN et al., 2009; KIMURA e KISHIMOTO, 2010), considerado um dos padrões de resposta imune responsáveis pela EAE. Diversos trabalhos discorrem sobre o importante papel da IL-6 na EAE (DIAB et al., 1997; SAMOILOVA et al., 1998; OKUDA et al., 1999; KORN et al., 2008; KORN et al., 2009; KIMURA e KISHIMOTO, 2010).

Em relação ao perfil de resposta envolvido na melhora da doença, a polarização da resposta imune para os perfis Th2 e T regulador está associado à melhora clínica da EAE por interferirem na proliferação de células pertencentes às populações Th1 e Th17, inibindo assim uma série de funções efetoras causadas por excessiva resposta inflamatória (BETTINI e VIGNALI, 2009). Nesse sentido, os resultados apresentados por Okuda et al. (1999) sugerem que a mudança de respostas de células Thl para Th2 poderia explicar a resistência dos camundongos deficientes para IL-6 à EAE. Isso porque, em comparação com as células provenientes do animal selvagem, as células do linfonodo de camundongos deficientes para IL-6 coletadas 12 dias após a imunização com o peptídeo 35-55 da glicoproteína da mielina de oligodendrócitos $\left(\mathrm{MOG}_{\mathrm{p} 35-55}\right)$ apresentaram uma produção maior de IL-4 e IL-10 e menor de IL-2 e IFN- $\gamma$ em resposta ao estímulo com a mesma proteína. Além disso, foi demonstrado que a transferência passiva de clones linfocitários produtores de IL-4, IL-10 e TGF- $\beta$ específicos ao antígeno MOG protegeram animais saudáveis da indução da EAE (CHEN et al., 1994; NICHOLSON et al., 1995; BROCKE et al., 1996). Em humanos também foi demonstrado o potencial protetor de populações produtoras destas citocinas durante a doença ativa (KIM e MOUDGIL, 2008; CORREALE e VILLA, 2010). Por esta razão, alternativas 
terapêuticas capazes de aumentar tais respostas seriam interessantes para amenizar ou suprimir os efeitos patogênicos ocasionados pelas populações Th1 e Th17 na EAE. 


\section{CONCLUSÕES}

Os resultados desse estudo mostram que os componentes salivares de A. aegypti apresentam um efeito imunomodulador em macrófagos peritoneais estimulados com LPS e IFN- $\gamma$, alterando o padrão de citocinas e a expressão de moléculas envolvidas com a ativação dessas células. Além disso, o EGS parece interferir negativamente na polarização de macrófagos para o perfil M1, mas não tem efeito no processo de diferenciação para o perfil M2. Ainda, uma proteína identificada na fração do EGS capaz de inibir significativamente a produção da citocina IL-6 por macrófagos ativados apresentou-se promissora no modelo de EAE, sendo capaz de alterar de maneira significativa o desenvolvimento da doença ao reduzir a polarização das respostas Th1 e Th17, sem afetar a polarização para o perfil Th2. 


\section{REFERÊNCIAS*}

ABREU, S. L. Suppression of experimental allergic encephalomyelitis by interferon. Immunol Commun, v. 11, n. 1, p. 1-7, 1982.

ALMEIDA, S. R. et al. Mouse B-1 cell-derived mononuclear phagocyte, a novel cellular component of acute non-specific inflammatory exudate. Int Immunol, v. 13, n. 9, p. 1193201, 2001.

ALMOLDA, B.; GONZALEZ, B.; CASTELLANO, B. Antigen presentation in EAE: role of microglia, macrophages and dendritic cells. Front Biosci (Landmark Ed), v. 16, p. 1157-71, 2011.

ARANGO DUQUE, G.; DESCOTEAUX, A. Macrophage cytokines: involvement in immunity and infectious diseases. Front Immunol, v. 5, p. 491, 2014.

BALDWIN, A. S. The NF-kappa B and I kappa B proteins: new discoveries and insights. Annu Rev Immunol, v. 14, p. 649-83, 1996.

BARROS, M. S. et al. Exposure to Aedes aegypti Bites Induces a Mixed-Type Allergic Response following Salivary Antigens Challenge in Mice. PLoS One, v. 11, n. 5, p. e0155454, 2016.

BASSO, A. S. et al. Reversal of axonal loss and disability in a mouse model of progressive multiple sclerosis. J Clin Invest, v. 118, n. 4, p. 1532-43, 2008.

BATOULIS, $\mathrm{H}$. et al. Experimental autoimmune encephalomyelitis-achievements and prospective advances. APMIS, v. 119, n. 12, p. 819-30, 2011.

BAXTER, A. G. The origin and application of experimental autoimmune encephalomyelitis. Nat Rev Immunol, v. 7, n. 11, p. 904-12, 2007.

BECHER, B.; SEGAL, B. M. T(H)17 cytokines in autoimmune neuro-inflammation. Curr Opin Immunol, v. 23, n. 6, p. 707-12, 2011.

BELL, J.; PATERSON, P. Y. Rapid induction of allergic encephalomyelitis in rats without the use of mycobacteria. Science, v. 131, n. 3411, p. 1448, 1960.

BEN-NUN, A.; WEKERLE, H.; COHEN, I. R. Vaccination against autoimmune encephalomyelitis with T-lymphocyte line cells reactive against myelin basic protein. Nature, v. 292 , n. 5818 , p. $60-1,1981$.

BERGER, T. et al. Experimental autoimmune encephalomyelitis: the antigen specificity of T lymphocytes determines the topography of lesions in the central and peripheral nervous system. Lab Invest, v. 76, n. 3, p. 355-64, 1997.

BETTINI, M.; VIGNALI, D. A. Regulatory T cells and inhibitory cytokines in autoimmunity. Curr Opin Immunol, v. 21, n. 6, p. 612-8, 2009. 
BIELEKOVA, B. et al. Regulatory CD56(bright) natural killer cells mediate immunomodulatory effects of IL-2Ralpha-targeted therapy (daclizumab) in multiple sclerosis. Proc Natl Acad Sci U S A, v. 103, n. 15, p. 5941-6, 2006.

Encephalitogenic potential of the myelin basic protein peptide (amino acids 83-99) in multiple sclerosis: results of a phase II clinical trial with an altered peptide ligand. Nat Med, v. 6, n. 10, p. 1167-75, 2000.

BILLIAU, A. et al. Enhancement of experimental allergic encephalomyelitis in mice by antibodies against IFN-gamma. J Immunol, v. 140, n. 5, p. 1506-10, 1988.

BISSONNETTE, E. Y.; ROSSIGNOL, P. A.; BEFUS, A. D. Extracts of mosquito salivary gland inhibit tumour necrosis factor alpha release from mast cells. Parasite Immunol, v. 15, n. 1, p. 27-33, 1993.

BIZZARRO, B. et al. Effects of Aedes aegypti salivary components on dendritic cell and lymphocyte biology. Parasit Vectors, v. 6, p. 329, 2013.

BOPPANA, V. D. et al. SAAG-4 is a novel mosquito salivary protein that programmes host CD4 T cells to express IL-4. Parasite Immunol, v. 31, n. 6, p. 287-95, 2009.

BORRA, R. C. et al. A simple method to measure cell viability in proliferation and cytotoxicity assays. Braz Oral Res, v. 23, n. 3, p. 255-62, 2009.

BROCKE, S. et al. Treatment of experimental encephalomyelitis with a peptide analogue of myelin basic protein. Nature, v. 379, n. 6563, p. 343-6, 1996.

BRONTE, V.; ZANOVELLO, P. Regulation of immune responses by L-arginine metabolism. Nat Rev Immunol, v. 5, n. 8, p. 641-54, 2005.

CALVO, E. et al. Aegyptin, a novel mosquito salivary gland protein, specifically binds to collagen and prevents its interaction with platelet glycoprotein VI, integrin alpha2beta1, and von Willebrand factor. J Biol Chem, v. 282, n. 37, p. 26928-38, 2007.

CARREGARO, V. et al. Nucleosides from Phlebotomus papatasi salivary gland ameliorate murine collagen-induced arthritis by impairing dendritic cell functions. J Immunol, v. 187, n. 8, p. 4347-59, 2011.

CHAMPAGNE, D. E.; RIBEIRO, J. M. Sialokinin I and II: vasodilatory tachykinins from the yellow fever mosquito Aedes aegypti. Proc Natl Acad Sci U S A, v. 91, n. 1, p. 138-42, 1994.

CHAMPAGNE, D. E. et al. The salivary gland-specific apyrase of the mosquito Aedes aegypti is a member of the 5'-nucleotidase family. Proc Natl Acad Sci U S A, v. 92, n. 3, p. 694-8, 1995.

CHASTAIN, E. M. et al. The role of antigen presenting cells in multiple sclerosis. Biochim Biophys Acta, v. 1812, n. 2, p. 265-74, 2011. 
CHEN, G. et al. Ixodes scapularis saliva mitigates inflammatory cytokine secretion during Anaplasma phagocytophilum stimulation of immune cells. Parasit Vectors, v. 5, p. 229, 2012.

CHEN, Y. et al. Regulatory $\mathrm{T}$ cell clones induced by oral tolerance: suppression of autoimmune encephalomyelitis. Science, v. 265, n. 5176, p. 1237-40, 1994.

CHU, C. Q.; WITTMER, S.; DALTON, D. K. Failure to suppress the expansion of the activated CD4 $\mathrm{T}$ cell population in interferon gamma-deficient mice leads to exacerbation of experimental autoimmune encephalomyelitis. J Exp Med, v. 192, n. 1, p. 123-8, 2000.

CHUNG, Y. et al. Critical regulation of early Th17 cell differentiation by interleukin-1 signaling. Immunity, v. 30, n. 4, p. 576-87, 2009.

COMPSTON, A.; COLES, A. Multiple sclerosis. Lancet, v. 372, n. 9648, p. 1502-17, 2008.

CONSTANTINESCU, C. S. et al. Antibodies against IL-12 prevent superantigen-induced and spontaneous relapses of experimental autoimmune encephalomyelitis. J Immunol, v. 161, n. 9, p. 5097-104, 1998.

CORRALIZA, I. M. et al. Determination of arginase activity in macrophages: a micromethod. J Immunol Methods, v. 174, n. 1-2, p. 231-5, 1994.

CORREALE, J.; VILLA, A. Role of $\mathrm{CD}^{+} \mathrm{CD} 25^{+} \mathrm{Foxp}^{+}$regulatory $\mathrm{T}$ cells in multiple sclerosis. Ann Neurol, v. 67, n. 5, p. 625-38, 2010.

CROSS, M. L.; CUPP, E. W.; ENRIQUEZ, F. J. Differential modulation of murine cellular immune responses by salivary gland extract of Aedes aegypti. Am J Trop Med Hyg, v. 51, n. 5, p. 690-6, 1994.

CUA, D. J. et al. Interleukin-23 rather than interleukin-12 is the critical cytokine for autoimmune inflammation of the brain. Nature, v. 421, n. 6924, p. 744-8, 2003.

DAVIS, M. J. et al. Macrophage M1/M2 polarization dynamically adapts to changes in cytokine microenvironments in Cryptococcus neoformans infection. MBio, v. 4, n. 3, p. e00264-13, 2013.

DIAB, A. et al. High IL-6 and low IL-10 in the central nervous system are associated with protracted relapsing EAE in DA rats. J Neuropathol Exp Neurol, v. 56, n. 6, p. 641-50, 1997.

DUONG, T. T. et al. Effect of anti-interferon-gamma monoclonal antibody treatment on the development of experimental allergic encephalomyelitis in resistant mouse strains. J Neuroimmunol, v. 53, n. 1, p. 101-7, 1994.

Effect of anti-interferon-gamma and anti-interleukin-2 monoclonal antibody treatment on the development of actively and passively induced experimental allergic encephalomyelitis in the SJL/J mouse. J Neuroimmunol, v. 36, n. 2-3, p. 105-15, $1992 .$. 
DÉRUAZ, M. et al. Ticks produce highly selective chemokine binding proteins with antiinflammatory activity. J Exp Med, v. 205, n. 9, p. 2019-31, 2008.

FERBER, I. A. et al. Mice with a disrupted IFN-gamma gene are susceptible to the induction of experimental autoimmune encephalomyelitis (EAE). J Immunol, v. 156, n. 1, p. 5-7, 1996.

FERREIRA, B. R.; SILVA, J. S. Saliva of Rhipicephalus sanguineus tick impairs T cell proliferation and IFN-gamma-induced macrophage microbicidal activity. Vet Immunol Immunopathol, v. 64, n. 3, p. 279-93, 1998.

FRANCISCHETTI, I. M. et al. The role of saliva in tick feeding. Front Biosci, v. 14, p. 2051-88, 2009.

FRAUENSCHUH, A. et al. Molecular cloning and characterization of a highly selective chemokine-binding protein from the tick Rhipicephalus sanguineus. J Biol Chem, v. 282, n. 37, p. 27250-8, 2007.

FROHMAN, E. M.; RACKE, M. K.; RAINE, C. S. Multiple sclerosis--the plaque and its pathogenesis. N Engl J Med, v. 354, n. 9, p. 942-55, 2006.

FUJIWARA, N.; KOBAYASHI, K. Macrophages in inflammation. Curr Drug Targets Inflamm Allergy, v. 4, n. 3, p. 281-6, 2005.

GHOSH, S.; MAY, M. J.; KOPP, E. B. NF-kappa B and Rel proteins: evolutionarily conserved mediators of immune responses. Annu Rev Immunol, v. 16, p. 225-60, 1998.

GHOSN, E. E. et al. Two physically, functionally, and developmentally distinct peritoneal macrophage subsets. Proc Natl Acad Sci U S A, v. 107, n. 6, p. 2568-73, 2010.

GILLESPIE, R. D. et al. Identification of an IL-2 binding protein in the saliva of the Lyme disease vector tick, Ixodes scapularis. J Immunol, v. 166, n. 7, p. 4319-26, 2001.

GONATAS, N. K.; HOWARD, J. C. Inhibition of experimental allergic encephalomyelitis in rats severely depleted of T cells. Science, v. 186, n. 4166, p. 839-41, 1974.

GORDON, S.; TAYLOR, P. R. Monocyte and macrophage heterogeneity. Nat Rev Immunol, v. 5, n. 12, p. 953-64, 2005.

GOVERMAN, J. Autoimmune T cell responses in the central nervous system. Nat Rev Immunol, v. 9, n. 6, p. 393-407, 2009.

GRAN, B. et al. IL-12p35-deficient mice are susceptible to experimental autoimmune encephalomyelitis: evidence for redundancy in the IL-12 system in the induction of central nervous system autoimmune demyelination. J Immunol, v. 169, n. 12, p. 7104-10, 2002.

GRETER, M. et al. Dendritic cells permit immune invasion of the CNS in an animal model of multiple sclerosis. Nat Med, v. 11, n. 3, p. 328-34, 2005.

GUILLIAMS, M. et al. Dendritic cells, monocytes and macrophages: a unified nomenclature based on ontogeny. Nat Rev Immunol, v. 14, n. 8, p. 571-8, 2014. 
HAJNICKÁ, V. et al. Anti-interleukin-8 activity of tick salivary gland extracts. Parasite Immunol, v. 23, n. 9, p. 483-9, 2001.

HARRINGTON, L. E. et al. Interleukin 17-producing CD4+ effector T cells develop via a lineage distinct from the T helper type 1 and 2 lineages. Nat Immunol, v. 6, n. 11, p. 1123$32,2005$.

HEMMER, B.; ARCHELOS, J. J.; HARTUNG, H. P. New concepts in the immunopathogenesis of multiple sclerosis. Nat Rev Neurosci, v. 3, n. 4, p. 291-301, 2002.

HIROTA, K. et al. Fate mapping of IL-17-producing T cells in inflammatory responses. Nat Immunol, v. 12, n. 3, p. 255-63, 2011.

HISCOTT, J.; KWON, H.; GÉNIN, P. Hostile takeovers: viral appropriation of the NFkappaB pathway. J Clin Invest, v. 107, n. 2, p. 143-51, 2001.

HOHLFELD, R.; WEKERLE, H. Immunological update on multiple sclerosis. Curr Opin Neurol, v. 14, n. 3, p. 299-304, 2001.

KAUR, G.; TROWSDALE, J.; FUGGER, L. Natural killer cells and their receptors in multiple sclerosis. Brain, v. 136, n. Pt 9, p. 2657-76, 2013.

KIM, E. Y.; MOUDGIL, K. D. Regulation of autoimmune inflammation by pro-inflammatory cytokines. Immunol Lett, v. 120, n. 1-2, p. 1-5, 2008.

KIMURA, A.; KISHIMOTO, T. IL-6: regulator of Treg/Th17 balance. Eur J Immunol, v. 40, n. 7, p. 1830-5, 2010.

KOJIMA, K. et al. Experimental autoimmune panencephalitis and uveoretinitis transferred to the Lewis rat by $\mathrm{T}$ lymphocytes specific for the $\mathrm{S} 100$ beta molecule, a calcium binding protein of astroglia. J Exp Med, v. 180, n. 3, p. 817-29, 1994.

KOMIYAMA, Y. et al. IL-17 plays an important role in the development of experimental autoimmune encephalomyelitis. J Immunol, v. 177, n. 1, p. 566-73, 2006.

KONÍK, P. et al. Anti-tumour necrosis factor-alpha activity in Ixodes ricinus saliva. Parasite Immunol, v. 28, n. 12, p. 649-56, 2006.

KORN, T. et al. IL-17 and Th17 Cells. Annu Rev Immunol, v. 27, p. 485-517, 2009.

IL-6 controls Th17 immunity in vivo by inhibiting the conversion of conventional T cells into Foxp3+ regulatory T cells. Proc Natl Acad Sci U S A, v. 105, n. 47, p. 18460-5, 2008.

KRAKOWSKI, M.; OWENS, T. Interferon-gamma confers resistance to experimental allergic encephalomyelitis. Eur J Immunol, v. 26, n. 7, p. 1641-6, 1996.

KUTHEJLOVÁ, M. et al. Tick salivary gland extract inhibits killing of Borrelia afzelii spirochetes by mouse macrophages. Infect Immun, v. 69, n. 1, p. 575-8, 2001. 
KÝCKOVÁ, K.; KOPECKÝ, J. Effect of tick saliva on mechanisms of innate immune response against Borrelia afzelii. J Med Entomol, v. 43, n. 6, p. 1208-14, Nov 2006.

LAGASSE, E.; WEISSMAN, I. L. Flow cytometric identification of murine neutrophils and monocytes. J Immunol Methods, v. 197, n. 1-2, p. 139-50, 1996.

LANGRISH, C. L. et al. IL-23 drives a pathogenic $\mathrm{T}$ cell population that induces autoimmune inflammation. J Exp Med, v. 201, n. 2, p. 233-40, 2005.

LEBOULLE, G. et al. Characterization of a novel salivary immunosuppressive protein from Ixodes ricinus ticks. J Biol Chem, v. 277, n. 12, p. 10083-9, 2002.

LEONARD, J. P.; WALDBURGER, K. E.; GOLDMAN, S. J. Prevention of experimental autoimmune encephalomyelitis by antibodies against interleukin 12. J Exp Med, v. 181, n. 1, p. 381-6, 1995.

LIN, Y. L. et al. Inhibition of Japanese encephalitis virus infection by nitric oxide: antiviral effect of nitric oxide on RNA virus replication. J Virol, v. 71, n. 7, p. 5227-35, 1997.

LINK, H.; HUANG, Y. M.; XIAO, B. G. Dendritic cells in experimental allergic encephalomyelitis and multiple sclerosis. J Neuroimmunol, v. 100, n. 1-2, p. 102-10, 1999.

LITZENBURGER, T. et al. B lymphocytes producing demyelinating autoantibodies: development and function in gene-targeted transgenic mice. J Exp Med, v. 188, n. 1, p. 16980, 1998.

LUBLIN, F. D. et al. Monoclonal anti-gamma interferon antibodies enhance experimental allergic encephalomyelitis. Autoimmunity, v. 16, n. 4, p. 267-74, 1993.

LUCCHINETTI, C. et al. Heterogeneity of multiple sclerosis lesions: implications for the pathogenesis of demyelination. Ann Neurol, v. 47, n. 6, p. 707-17, 2000.

MACKANESS, G. B. Cellular resistance to infection. J Exp Med, v. 116, p. 381-406, 1962. $\overline{20,1964 .}$

The immunological basis of acquired cellular resistance. J Exp Med, v. 120, p. 105-

MANGIARDI, M. et al. An animal model of cortical and callosal pathology in multiple sclerosis. Brain Pathol, v. 21, n. 3, p. 263-78, 2011.

MCCARTHY, D. P.; RICHARDS, M. H.; MILLER, S. D. Mouse models of multiple sclerosis: experimental autoimmune encephalomyelitis and Theiler's virus-induced demyelinating disease. Methods Mol Biol, v. 900, p. 381-401, 2012.

MCKIEL, J. Sensitization to mosquito bites. Can. J. Zool., v. 37, p. 11, 1959.

MEDZHITOV, R. Origin and physiological roles of inflammation. Nature, v. 454, n. 7203, p. 428-35, 2008. 
MERRILL, J. E. et al. Inflammatory leukocytes and cytokines in the peptide-induced disease of experimental allergic encephalomyelitis in SJL and B10.PL mice. Proc Natl Acad Sci U S A, v. 89, n. 2, p. 574-8, 1992.

MINISTÉRIO-DA-SAÚDE. Protocolo clínico e Diretrizes terapêuticas esclerose múltipla. Disponível em: http://portalarquivos.saude.gov.br/images/pdf/2015/maio/06/PCDTEsclerose-Multipla-06-05-2015.pdf. 2015.

MOLINÉ-VELÁZQUEZ, V. et al. Myeloid cell distribution and activity in multiple sclerosis. Histol Histopathol, p. 11699, 2015.

MOSSER, D. M.; EDWARDS, J. P. Exploring the full spectrum of macrophage activation. Nat Rev Immunol, v. 8, n. 12, p. 958-69, 2008.

MUNDER, M.; EICHMANN, K.; MODOLELL, M. Alternative metabolic states in murine macrophages reflected by the nitric oxide synthase/arginase balance: competitive regulation by $\mathrm{CD}^{+} \mathrm{T}$ cells correlates with Th1/Th2 phenotype. J Immunol, v. 160, n. 11, p. 5347-54, 1998.

MURRAY, P. J. et al. Macrophage activation and polarization: nomenclature and experimental guidelines. Immunity, v. 41, n. 1, p. 14-20, 2014.

MURRAY, P. J.; WYNN, T. A. Protective and pathogenic functions of macrophage subsets. Nat Rev Immunol, v. 11, n. 11, p. 723-37, 2011.

MÄ̈̈TTё, J. A. et al. Encephalitogenicity of myelin-associated oligodendrocytic basic protein and 2',3'-cyclic nucleotide 3'-phosphodiesterase for BALB/c and SJL mice. Immunology, v. 95, n. 3, p. 383-8, 1998.

NATHAN, C. F.; KARNOVSKY, M. L.; DAVID, J. R. Alterations of macrophage functions by mediators from lymphocytes. J Exp Med, v. 133, n. 6, p. 1356-76, 1971.

NATHAN, C. F. et al. Identification of interferon-gamma as the lymphokine that activates human macrophage oxidative metabolism and antimicrobial activity. J Exp Med, v. 158, n. 3, p. $670-89,1983$.

NICHOLSON, L. B. et al. An altered peptide ligand mediates immune deviation and prevents autoimmune encephalomyelitis. Immunity, v. 3, n. 4, p. 397-405, 1995.

NORSWORTHY, N. B. et al. Sand fly saliva enhances Leishmania amazonensis infection by modulating interleukin-10 production. Infect Immun, v. 72, n. 3, p. 1240-7, 2004.

O'CONNOR, R. A. et al. Cutting edge: Th1 cells facilitate the entry of Th17 cells to the central nervous system during experimental autoimmune encephalomyelitis. J Immunol, v. 181, n. 6, p. 3750-4, 2008.

OKUDA, Y. et al. IL-6 plays a crucial role in the induction phase of myelin oligodendrocyte glucoprotein 35-55 induced experimental autoimmune encephalomyelitis. J Neuroimmunol, v. 101, n. 2, p. 188-96, 1999. 
OLIVEIRA, C. J. et al. Deconstructing tick saliva: non-protein molecules with potent immunomodulatory properties. J Biol Chem, v. 286, n. 13, p. 10960-9, 2011.

OPPMANN, B. et al. Novel p19 protein engages IL-12p40 to form a cytokine, IL-23, with biological activities similar as well as distinct from IL-12. Immunity, v. 13, n. 5, p. 715-25, 2000 .

ORTIZ-ORTIZ, L.; WEIGLE, W. O. Cellular events in the induction of experimental allergic encephalomyelitis in rats. J Exp Med, v. 144, n. 3, p. 604-16, 1976.

PARK, $\mathrm{H}$. et al. A distinct lineage of CD4 $\mathrm{T}$ cells regulates tissue inflammation by producing interleukin 17. Nat Immunol, v. 6, n. 11, p. 1133-41, 2005.

PIETRANGELO, A.; HIGUERA, V. Multiple Sclerosis by the Numbers: Facts, Statistics, and You. Healthline. Disponível em: <http://www.healthline.com/health/multiple-sclerosis/factsstatistics-infographic>, 2015.

PLOWDEN, J. et al. Innate immunity in aging: impact on macrophage function. Aging Cell, v. 3, n. 4, p. 161-7, 2004.

PRINEAS, J. W.; PARRATT, J. D. Oligodendrocytes and the early multiple sclerosis lesion. Ann Neurol, v. 72, n. 1, p. 18-31, 2012.

RAMOS, A. Imunomodulação da encefalomielite autoimune experimental pelo extrato da glândula salivar de Aedes aegypti. Universidade de São Paulo, 2014. 107.

RAY, A.; DITTEL, B. N. Isolation of mouse peritoneal cavity cells. J Vis Exp, n. 35, 2010.

REUNALA, T. et al. Immunology and treatment of mosquito bites. Clin Exp Allergy, v. 20 Suppl 4, p. 19-24, 1990.

RIBEIRO, J. M. Role of saliva in blood-feeding by arthropods. Annu Rev Entomol, v. 32, p. 463-78, 1987.

Characterization of a vasodilator from the salivary glands of the yellow fever mosquito Aedes aegypti. J Exp Biol, v. 165, p. 61-71, 1992.

Blood-feeding in mosquitoes: probing time and salivary gland anti-haemostatic activities in representatives of three genera (Aedes, Anopheles, Culex). Med Vet Entomol, v. 14, n. 2, p. 142-8, 2000.

RIBEIRO, J. M. et al. Salivary apyrase of Aedes aegypti: characterization and secretory fate. Comp Biochem Physiol B, v. 79, n. 1, p. 81-6, 1984.

RIBEIRO, J. M. C.; ASSUMPÇÃO, T. C.; FRANCISCHETTI, M. B. An Insight into the Sialomes of Bloodsucking Heteroptera. Psyche, p.16. 2012

ROBINSON, A. P. et al. The experimental autoimmune encephalomyelitis (EAE) model of MS: utility for understanding disease pathophysiology and treatment. Handb Clin Neurol, v. 122, p. 173-89, 2014. 
SAMOILOVA, E. B. et al. IL-6-deficient mice are resistant to experimental autoimmune encephalomyelitis: roles of IL-6 in the activation and differentiation of autoreactive T cells. $\mathbf{J}$ Immunol, v. 161, n. 12, p. 6480-6, 1998.

SCHNEIDER, B. S. et al. Aedes aegypti saliva alters leukocyte recruitment and cytokine signaling by antigen-presenting cells during West Nile virus infection. PLoS One, v. 5, n. 7, p. e11704, 2010.

Aedes aegypti salivary gland extracts modulate anti-viral and TH1/TH2 cytokine responses to sindbis virus infection. Viral Immunol, v. 17, n. 4, p. 565-73, 2004.

SEGAL, B. M.; DWYER, B. K.; SHEVACH, E. M. An interleukin (IL)-10/IL-12 immunoregulatory circuit controls susceptibility to autoimmune disease. J Exp Med, v. 187, n. 4, p. 537-46, 1998.

SIEWEKE, M. H.; ALLEN, J. E. Beyond stem cells: self-renewal of differentiated macrophages. Science, v. 342, n. 6161, p. 1242974, 2013.

SOARES, M. B. et al. The vasoactive peptide maxadilan from sand fly saliva inhibits TNFalpha and induces IL- 6 by mouse macrophages through interaction with the pituitary adenylate cyclase-activating polypeptide (PACAP) receptor. J Immunol, v. 160, n. 4, p. 1811-6, 1998.

STARK, K. R.; JAMES, A. A. A factor Xa-directed anticoagulant from the salivary glands of the yellow fever mosquito Aedes aegypti. Exp Parasitol, v. 81, n. 3, p. 321-31, 1995.

Isolation and characterization of the gene encoding a novel factor Xa-directed anticoagulant from the yellow fever mosquito, Aedes aegypti. J Biol Chem, v. 273, n. 33, p. 20802-9, 1998.

STEINMAN, L. A brief history of $T(H) 17$, the first major revision in the $T(H) 1 / T(H) 2$ hypothesis of T cell-mediated tissue damage. Nat Med, v. 13, n. 2, p. 139-45, 2007.

SÁ-NUNES, A. et al. The immunomodulatory action of sialostatin $\mathrm{L}$ on dendritic cells reveals its potential to interfere with autoimmunity. J Immunol, v. 182, n. 12, p. 7422-9, 2009.

Prostaglandin E2 is a major inhibitor of dendritic cell maturation and function in Ixodes scapularis saliva. J Immunol, v. 179, n. 3, p. 1497-505, 2007.

SÁ-NUNES, A.; OLIVEIRA, C. Sialogenins and other immunomodulators from blood feeding. In: KINI, R.;MCLANE, M., et al. (Ed.). Toxins and Hemostasis: From Bench to Beside: Springer, 2009. p.131-152.

TAYLOR, P. R. et al. Macrophage receptors and immune recognition. Annu Rev Immunol, v. 23, p. 901-44, 2005.

TEIXEIRA, C. R. et al. Saliva from Lutzomyia longipalpis induces CC chemokine ligand $2 /$ monocyte chemoattractant protein-1 expression and macrophage recruitment. J Immunol, v. 175, n. 12, p. 8346-53, 2005. 
THEOBALDO, M. et al. IL-10 produced by B1 cells modulates inflammatory response to LPS. Faseb Journal, 2010.

THEODOS, C. M.; TITUS, R. G. Salivary gland material from the sand fly Lutzomyia longipalpis has an inhibitory effect on macrophage function in vitro. Parasite Immunol, v. 15, n. 8, p. 481-7, 1993.

THOUA, N. M. et al. Encephalitogenic and immunogenic potential of the stress protein alphaB-crystallin in Biozzi ABH (H-2A(g7)) mice. J Neuroimmunol, v. 104, n. 1, p. 47-57, 2000.

TSIKAS, D. Analysis of nitrite and nitrate in biological fluids by assays based on the Griess reaction: appraisal of the Griess reaction in the L-arginine/nitric oxide area of research. $\mathbf{J}$ Chromatogr B Analyt Technol Biomed Life Sci, v. 851, n. 1-2, p. 51-70, 2007.

TULLMAN, M. J. Overview of the epidemiology, diagnosis, and disease progression associated with multiple sclerosis. Am J Manag Care, v. 19, n. 2 Suppl, p. S15-20, 2013.

UEHARA, E. U.; SHIDA, B. E. S.; DE BRITO, C. A. Role of nitric oxide in immune responses against viruses: beyond microbicidal activity. Inflamm Res, v. 64, n. 11, p. 845-52, 2015.

VAN DYKEN, S. J.; LOCKSLEY, R. M. Interleukin-4- and interleukin-13-mediated alternatively activated macrophages: roles in homeostasis and disease. Annu Rev Immunol, v. 31, p. 317-43, 2013.

VAN FURTH, R.; COHN, Z. A. The origin and kinetics of mononuclear phagocytes. J Exp Med, v. 128, n. 3, p. 415-35, 1968.

VARADHACHARY, A. S.; MONESTIER, M.; SALGAME, P. Reciprocal induction of IL10 and IL-12 from macrophages by low-density lipoprotein and its oxidized forms. Cell Immunol, v. 213, n. 1, p. 45-51, 2001.

VERMES, I. et al. A novel assay for apoptosis. Flow cytometric detection of phosphatidylserine expression on early apoptotic cells using fluorescein labelled Annexin V. J Immunol Methods, v. 184, n. 1, p. 39-51, 1995.

VOORTHUIS, J. A. et al. Suppression of experimental allergic encephalomyelitis by intraventricular administration of interferon-gamma in Lewis rats. Clin Exp Immunol, v. 81, n. 2, p. 183-8, 1990.

WAITUMBI, J.; WARBURG, A. Phlebotomus papatasi saliva inhibits protein phosphatase activity and nitric oxide production by murine macrophages. Infect Immun, v. 66, n. 4, p. 1534-7, 1998.

WAKSMAN, B. H.; ARBOUYS, S.; ARNASON, B. G. The use of specific "lymphocyte" antisera to inhibit hypersensitive reactions of the "delayed" type. J Exp Med, v. 114, p. 997$1022,1961$. 
WANASEN, N. et al. Differential modulation of murine host immune response by salivary gland extracts from the mosquitoes Aedes aegypti and Culex quinquefasciatus. Med Vet Entomol, v. 18, n. 2, p. 191-9, 2004.

WASSERMAN, H. A. Modulation of mammalian immune effector cell functions by saliva of yellow fever mosquito, Aedes aegypti (Diptera: Culicidae). 2005. 168 (Dissertation). University of Georgia, Athens, Georgia.

WASSERMAN, H. A.; SINGH, S.; CHAMPAGNE, D. E. Saliva of the Yellow Fever mosquito, Aedes aegypti, modulates murine lymphocyte function. Parasite Immunol, v. 26, n. 6-7, p. 295-306, 2004.

WEKERLE, H. et al. Autoimmunity's next top models. Nat Med, v. 18, n. 1, p. 66-70, 2012.

WILLENBORG, D. O. et al. IFN-gamma plays a critical down-regulatory role in the induction and effector phase of myelin oligodendrocyte glycoprotein-induced autoimmune encephalomyelitis. J Immunol, v. 157, n. 8, p. 3223-7, 1996.

WUEST, S. C. et al. A role for interleukin-2 trans-presentation in dendritic cell-mediated T cell activation in humans, as revealed by daclizumab therapy. Nat Med, v. 17, n. 5, p. 604-9, 2011.

YONA, S.; GORDON, S. From the Reticuloendothelial to Mononuclear Phagocyte System The Unaccounted Years. Front Immunol, v. 6, p. 328, 2015.

ZAMVIL, S. S.; STEINMAN, L. The $\mathrm{T}$ lymphocyte in experimental allergic encephalomyelitis. Annu Rev Immunol, v. 8, p. 579-621, 1990.

ZEIDNER, N. S. et al. Mosquito feeding modulates Th1 and Th2 cytokines in flavivirus susceptible mice: an effect mimicked by injection of sialokinins, but not demonstrated in flavivirus resistant mice. Parasite Immunol, v. 21, n. 1, p. 35-44, 1999.

ZHANG, G. et al. Early detection of apoptosis using a fluorescent conjugate of annexin V. Biotechniques, v. 23, n. 3, p. 525-31, 1997.

ZHANG, G. X. et al. Induction of experimental autoimmune encephalomyelitis in IL-12 receptor-beta 2-deficient mice: IL-12 responsiveness is not required in the pathogenesis of inflammatory demyelination in the central nervous system. J Immunol, v. 170, n. 4, p. 215360, 2003.

ZHANG, X. Regulatory functions of innate-like B cells. Cell Mol Immunol, v. 10, n. 2, p. 113-21, 2013.

ZHANG, X.; GONCALVES, R.; MOSSER, D. M. The isolation and characterization of murine macrophages. Curr Protoc Immunol, v. Chapter 14, p. Unit 14.1, 2008. 